\title{
6 Plessners Freud-Kritiken. Zum Verhältnis zwischen Philosophischer Anthropologie und klassischer Psychoanalyse
}

\section{1 Überblick}

Im vorigen, dem 5. Kapitel wurde das Rahmenwerk einer interdisziplinären Wissenschaft von der Personalität vorgestellt. Hat man dieses vertikalhorizontale framework für die Lebensführung von Personen vor Augen, versteht man leicht den systematischen Gehalt und Zusammenhang von Plessners Stellungnahmen $\mathrm{zu}$ anderen Forschungsrichtungen, so auch die $\mathrm{zu}$ Sigmund Freuds Psychoanalyse. Dabei greife ich im Folgenden die vier wichtigsten Stellungnahmen von Helmuth Plessner in seiner Philosophischen Anthropologie zur klassischen Psychoanalyse heraus. Leider sind sie bisher in der FreudForschung (vgl. Lohmann/Pfeiffer 2013) überhaupt nicht zur Kenntnis genommen und in der Plessner-Forschung nie systematisch und im Ganzen behandelt worden.

Die ersten beiden Stellungnahmen erfolgen von Plessner unter seinem vertikalen Aspekt der naturphilosophischen Vergleichbarkeit von Menschen und Anthropoiden. Sie betreffen in Freuds Werk dessen naturwissenschaftlich orientierten Anspruch auf eine kausale Erklärung von Phänomenen des Menschseins. Ich beginne unter 6.2. mit Plessners Kritik an der klassischen Psychoanalyse in dem Buch Die Stufen des Organischen und der Mensch von 1928, d. h. in der naturphilosophischen Fundierung der Philosophischen Anthropologie. Darauf folgt unter 6.3. Plessners Stellungnahme in seiner geschichtsanthropologischen Abhandlung Zur Frage nach der Conditio humana (1961, hier Plessner 1983e), die als Einleitung in die Propyläen Weltgeschichte von Golo Mann und Alfred Heuß erschienen ist. Hier verweist Plessner systematisch auf Freud nur, insofern man das Anthropoide im Vergleich mit der Humanontogenese und phylogenese als Ermöglichung von Geschichte braucht.

Sodann gehe ich auf die beiden wichtigsten Bezugnahmen auf Freud unter Plessners horizontalem Aspekt des geschichtlichen Verstehens interpersonaler Relationen zwischen Personen ein, die in ihren Körper-Leib-Differenzen leben. Hier nimmt Plessner Freud als einen Hermeneutiker der zwischenmenschlichen Phänomene des Witzes und des Unheimlichen in der conditio humana ernst, deren Verstehensleistung von seinem kausalen Erklärungsanspruch befreit werden kann. In dieser Perspektive interessiert zunächst unter 6.4. Plessners Einschätzung von Freuds Beitrag zum Verstehen der ,Witzigkeit‘. Plessner 
kommt in seinem Buch Lachen und Weinen. Eine Untersuchung der Grenzen menschlichen Verhaltens (1941, hier Plessner 1982f) auch auf den Witz als einen der vielen Anlässe zum Lachen und damit auf Freud zu sprechen. Schließlich gehe ich unter 6.5. Plessners Rückgriff auf Freuds Verständnis des Unheimlichen nach, der sich in Plessners Buch Macht und menschliche Natur. Ein Versuch zur Anthropologie der geschichtlichen Weltansicht (1931, hier Plessner 1981d) findet.

\subsection{Zu Plessners Einordnung und Kritik an der Psychoanalyse in seinen Stufen des Organischen und der Mensch (1928)}

Im ersten Schritt seiner Auseinandersetzung mit der Psychoanalyse räumt Plessner ein, dass die „Verdrängung und Sublimierung“ von Trieben „wichtige“ Phänomene darstellen (Plessner 1975, 38). Diese qualitativen Erfahrungen seien wichtig in der Ontogenese und Phylogenese von Menschen und Anthropoiden im Vergleich. Es sei aber oft so, dass die Erforscher eines Phänomens die Wichtigkeit ihres Gegenstandsbereichs mit der Rolle des Fundaments für die Wissenschaft verwechseln und dadurch in einen schlechten Zirkel geraten:

Etwas kann sehr wichtig für die Entwicklung unserer Einsichten sein, fundamental wichtig, wie man sagt, ohne gleich den Charakter eines echten Fundaments zu haben. Echtes Fundament trägt, ohne selbst getragen zu sein. Der Empiriker achtet pflichtgemäß nur auf die erste Funktion. (Ebd.)

Der Empiriker achtet also auf die Wichtigkeit des Phänomens z. B. des Unbewussten für die Entwicklung unserer Einsicht, dass der Mensch nicht einfach ein animal rationale sei. Der Philosoph müsse indessen nach der zweiten Funktion fragen, die die begriffliche und methodische Verbindung des Forschungsverfahrens zum Gegenstandsbereich freilegt und herstellt (siehe ebd.).

Daraus ergibt sich für den Gegenstandsbereich einer vergleichenden Genese die Frage, ob er tatsächlich durch ein triebdynamisches Modell vollständig zu erfassen sei, in dem dann höchstens die Verdrängung und Sublimierung von Trieben erwartet werden kann. Durch dieses „Objektprinzip“ (ebd.) sei der Gegenstandsbereich bereits festgelegt. Auch für das Forschungsverfahren sei es nicht selbstverständlich, sondern fraglich, ob es selber allein auf dem Niveau der Verdrängung und Sublimierung von Trieben stattfinden könne. Führt dieses Niveau tatsächlich zu wissenschaftlicher Erkenntnis oder ist es nur darauf ausgerichtet, einseitiges Mitmachen in wechselseitiges Mitmachen der Verdrängung und Sublimierung fortzusetzen? Die Konfusionsprobleme zwischen Pati- 
entInnen und TherapeutInnen während triebdynamischer Therapien sind wohl bekannt. Plessner sieht erst in den vertikalen und horizontalen Relationen von Personalität den nötigen geistigen Abstand zur Triebdynamik, den die exzentrische Positionalität gewähre, damit sich nicht die beteiligten Personen nur ihre Verdrängungen und Sublimierungen gegenseitig vorrechnen müssten.

Im zweiten Schritt seiner Auseinandersetzung mit der Psychoanalyse ordnet Plessner die triebdynamischen Modelle grundsätzlich der Sphäre von Tieren zu, d. h. als dem Gegenstande nach gerichtet auf dasjenige, was er kategorial eine geschlossene Organisationsform in einer zentrischen Positionalitätsform nennt. „In seiner Selbständigkeit ist das Tier Ausgangspunkt und Angriffspunkt seiner Triebe, die nichts anderes als unmittelbare Manifestationen der primären Unerfülltheit“ sind und damit „die mittelbare Eingliederung in den Lebenskreis bedeuten“ (Plessner 1975, 233). Der Neurophysis, in der das Gehirn eine zentralnervöse Schließung der Organisationsform darstellt, entspreche im Verhalten, d. h. im Sich-Positionieren des Lebewesens, dass sein Leib die Verbindung zwischen dem Organismus und etwas in der Umwelt übernimmt. Die triebdynamische Interpretation des tierlichen Verhaltens setze an dem Leib des Tieres an, dessen Getriebensein, aus der „Unerfülltheit“ in die „Erfüllung“ (ebd.) zu gelangen. Für die Erfüllung von Trieben gebe es dann ein Spektrum, das von Instinkten, deren Auslösung starr festgelegt ist, bis zur intelligent erlernten Erfüllung von Trieben reiche, wie es sich in Wolfgang Köhlers Nachweis der Intelligenz von Schimpansen bewährt habe. Die triebdynamischen Ansätze enthalten bereits Intelligenz, d. h. die plötzliche Einsicht in die Lösung eines situativen Problems, ohne dass diese Einsicht zuvor in der Population oder der Lebensgeschichte des Tieres aufgetaucht wäre. Es ging bei diesen Schimpansenversuchen nicht nur um die Umfunktionierung von Vorgefundenem in ein Werkzeug, sondern auch bereits um die Herstellung von Werkzeugen für die Erfüllung des Ernährungstriebes. Die Schimpansen waren durchaus dazu in der Lage, Triebzustände so aufzuschieben, dass sie auf dem Umweg einer intelligenten Lösung dann doch zur Trieberfüllung kamen (ebd., 266-270).

Was sollte dies nun für eine vergleichende Untersuchung der Ontogenese von Menschen und Anthropoiden bedeuten? Wolfgang Köhler blieb klar im Rahmen der Biologie, d. h. die biologischen Verhaltensfunktionen gaben die Erfüllungsziele der Triebe vor (Köhler 1917). Sein biologischer Erkenntnisanspruch war darauf begrenzt, dass andere das konzeptionelle und methodische Arrangement seiner Laborversuche öffentlich reproduzieren und so objektivieren können. Im Falle der Psychoanalyse schien alles doppeldeutig zu sein. Freud schien einerseits einem neurobiologischen und evolutionsbiologischen Erklärungsanspruch verpflichtet, andererseits aber in der Diskussion mit Ande- 
ren immer stärker einen geisteswissenschaftlichen und kulturgeschichtlichen Verstehensanspruch zu entwickeln, auf den ich unter 6.4. und 6.5. zurückkomme. Die Verlängerung des tierpsychologischen Trieb-Modells (Freud 1999e) in die Relation zwischen Ich, Es und Über-Ich (Freud 1999a) wurde im Laufe der Entwicklung der Psychoanalyse immer doppeldeutiger (umso mehr, wenn man Alfred Adler berücksichtigte). 1928 aber stand für Plessner deutlich der naturalistische Erklärungsanspruch der Psychoanalyse als medizinischer Therapie im Vordergrund, ihrer Selbstdarstellung als anerkannter wissenschaftlicher Beruf entsprechend.

Im dritten Schritt seiner Auseinandersetzung mit der Psychoanalyse ordnet Plessner ihren naturalistischen Erklärungsanspruch ein. Da sich dieser Erklärungsanspruch auf den Menschen bezieht, erörtert ihn Plessner im Abschlusskapitel seiner Stufen über die exzentrische Positionalität. Sein eigener Anspruch besteht nicht darin, eine kausale Erklärung der Humanontogenese und Humanphylogenese leisten zu können, worum es auch der Psychoanalyse letztlich nicht gehe, da sie Lebensprobleme von Personen in der heutigen bürgerlichen Zivilisation voraussetze und ihre Therapien darauf beziehe, allerdings ohne dies reflexiv einzuholen. Plessners eigener Anspruch richtet sich darauf, diese gemeinsamen lebensweltlichen Voraussetzungen als die Ermöglichungsstrukturen personalen Lebens zu explizieren.

Uns Personen falle in der Deutung der Schimpansen-Intelligenz auf, dass sie sich nicht aus dem Triebgefüge des Einzeltiers und aus dem Mitmachen des einen mit dem anderen Tier ablösen und nicht von einer Situationsart auf eine andere Situationsart übertragen lasse. Wo wir Personen wie selbstverständlich eine Transponierbarkeit der intelligenten Einsicht in einen Strukturzusammenhang von Situationsart zu Situationsart annehmen und diesen auch durch Symbolfunktionen im Hinblick auf Irreales verwirklichen können, bleibe die Schimpansenintelligenz auf ein Gedächtnis für senso-motorisch mögliche Ringschlüsse im zentrischen Lebenskreis angewiesen. Deren psychisches Korrelat bestehe in der Vorstellung von Feldverhalten, nicht aber in der Ablösung von Sachverhalten. Die Schimpansen-Intelligenz arbeite nicht mit spezifisch geistigen Kontrasten von Abwesenheit zur Beurteilung des sinnlich Anwesenden. Die Werkzeuge können z. B. nicht nach einem Gravitationsgesetz, das einen leeren Raum und eine leere Zeit voraussetzt, miteinander verbunden werden, sondern lediglich nach den leiblichen Aktivitätspotentialen und deren leiblichem Gedächtnis. Demgegenüber nehmen Personen geistige Orientierungen in Anspruch, die aus dem Nichts der physikalischen Raumzeit und aus dem Nirgendwo und Nirgendwann der leiblichen Erfüllung hier und jetzt stammen (siehe Plessner 1975, 270-277, 284-287). Personen nehmen geistig an einer Mitwelt teil, die außer- 
halb, d. h. exzentrisch, von der Außen- und Innenwelt der Körper und Leiber steht. Gleichwohl bleiben Personen als Lebewesen darauf angewiesen, dass sie ihre geistigen Orientierungen in der Welt physikalischer Körper und in der Welt eigenräumlicher und eigenzeitlicher Leiber verwirklichen können, mithin ihre Exzentrizität auch konzentrisch vollziehen. Sie bleiben Lebewesen. Personen stehen so in diesem Hiatus zwischen einerseits einer geistig geteilten Mitwelt und andererseits der Welt der Körper, die für sie reversibel sind, und der Leiber, die für sie irreversibel sind (ebd., 293-294). Daher stehen Personen schon immer in einer Aufgabe, nämlich der, diesen Hiatus im Vollzug verschränken zu können, mithin auf die Fraglichkeit ihres Bruches geschichtlich zu antworten (ebd., 292, 309-311, 337-339).

Ich habe deshalb diese Philosophische Anthropologie eine der Negativität des Absoluten genannt (Krüger 1999). Die Exzentrizität der Positionalität gründet auf dem Nichts, Nirgendwann und Nirgendwo (Plessner 1975, 293, 341-346). Diese Philosophische Anthropologie von Plessner ist weder essentialistisch noch anti-essentialistisch, worum sich leider auf unproduktive Weise die heutige Diskussion seit Jahrzehnten dreht. Laut Plessners Philosophischer Anthropologie besteht das Wesen der personalen Lebenssphäre in dem Hiatus zwischen der Exzentrizität und der Positionalität. Daher existiere personales Leben in einer offenen Fraglichkeit, auf die es nur im geschichtlichen Prozess und daher nicht abschließend antworten kann. Die Geschichtlichkeit wurzelt in dem Bruch und ist mithin nicht überwindbar. Es gibt keine Aufhebung in ein höheres und positives Absolutes außer im Utopischen.

Mit der exzentrischen Positionalität als der wirklichen Ermöglichungsstruktur der personalen Lebenssphäre, so Plessner, werde systematisch einsehbar und verstehbar, wozu die Humanontogenese und die Humanphylogenese bislang geführt haben. So falsch es wäre, diese Genesen teleologisch aus dem Geist erklären $\mathrm{zu}$ wollen (ein spiritualistischer Fehlschluss), so falsch wäre es aus Plessners Sicht ebenso, diese Genesen allein aus einem Triebmodell erklären zu wollen, das bei Anthropoiden zwar greife, aber an dem Wohin der beiden Genesen zur Personalität scheitere. Dem spiritualistischen Fehlschluss entspreche also umgekehrt ein naturalistischer Fehlschluss, als ob wir Personen nicht bereits auf Abstand unsere natürliche Gewordenheit thematisieren könnten. Die personale Lebenssphäre sei von Natur, d. h. aus ihrem Bruch heraus, künstlich (ebd., 310). Sie habe keinen anderen Zugang zur Natur als den durch Kultur und Artefakte in ihrer eigenen Geschichte, d. h. keinen anderen Kontakt zur Unmittelbarkeit der Natur als den durch Vermittlungen hindurch, die geschichtlich habitualisiert werden und daher als natürlich im geschichtlichen Sinne, nicht im Sinne einer Kausalanalyse, erscheinen. 
Gemessen an dem Wozu der Humanontogenese und -phylogenese, d. h. an der Personalität in der Mitwelt, ordnet Plessner nun die Psychoanalyse in eine negative und indirekte Modifikation der naturalistischen Erklärungsversuche von Kultur ein. Die positiven Modifikationen der naturalistischen Erklärung, so in der Evolutionstheorie, sehen in der menschlichen Kultur einen begrüßenswerten Fortschritt der allgemein in der Natur angelegten „Lebenserhaltung und Lebensförderung“ (ebd., 313), etwa anhand der Werkzeugherstellung, der Hirnentwicklung oder der wachsenden Nützlichkeit. Hinter ihrer Bejahung des ,Kampfs ums Dasein', durch Anpassung und Selektion in der Natur, stehe die Bejahung des industriegesellschaftlichen Fortschritts durch kapitalistische Konkurrenz. Die negative Modifikation der naturalistischen Erklärung sehe demgegenüber in der menschlichen Kulturentwicklung ein Problem der Erkrankung, weil ein Gleichgewicht zwischen Organismus und Umwelt durch die Erfüllung von Trieben als natürlich unterstellt werde. Dadurch erscheine der Bruch in der menschlichen Existenz als ein Ungleichgewicht, das in Pathologien führe. Unter dem Druck der Kultur müsse der Mensch demnach das „Unpassende ins Unterbewusstsein verdrängen“ (ebd.). Daraus resultierten zwei Formen der Triebentladung, die „Neurose“ und die „Sublimierung, d. h. die Umbiegung ins Geistige“ (ebd., 314). Die Triebsublimierung bleibe „eine indirekte Erfüllung der an ihrer direkten Sättigung gehemmten Triebregungen“ (ebd.). Freud und Adler erklärten die Hypertrophie der menschlichen Triebdynamik auf indirektem Wege ,unter der Idee der Flucht vor dem Trieb oder der Überkompensation“ (ebd.). „Für die negative Modifikation ist Kultur der indirekte Ausdruck des zur Selbststeigerung verdammten und vor ihr Rettung suchenden Lebens" (ebd. 315).

So werde aber nicht einsehbar und verständlich, worin der ,außernützliche Sinn“ kultureller Betätigungen liege, worin „das Eigengewicht der geistigen Sphäre“ bestehen könne, das aber doch die Psychoanalyse für sich selber als geistige Unternehmung in Anspruch nehme. Der „Kardinalfehler“ bestehe in der Annahme, nicht selber in geistigen Äußerungen und Gehalten tätig zu werden, sondern außerhalb des Geistes zu stehen, wodurch man vermeine, den Geist als „Epiphänomen“ aus „biologischen Prozessen herleiten“ zu können (alle Zitate ebd.). Die Objektivierung wissenschaftlicher Erkenntnis erfolge jedoch nicht in einer Natur außerhalb von Gesellschaft und Kultur, sondern in der Öffentlichkeitssphäre im Unterschied zur Privatsphäre von Gesellschaft und Kultur. Die Mitwelt ist an den öffentlichen Realisierungsmodus gebunden (ebd., 345). Die Psychoanalyse sei in einem schlechten Zirkel mit psychischen Symptomen beschäftigt, wenn sie diese für das Fundament einer Kausalanalyse der menschlichen Kultur halte. Sie könnte indessen aus diesen Symptomen in die 
„exzentrische Lebensstruktur“ heraustreten, um auf diesem Fundament die Symptome durch geistigen Abstand zu thematisieren und zu therapieren. Stattdessen trage die Psychoanalyse durch ihre kausale Rückführung der Kultur auf „Überkompensation oder Sublimierung auf Grund von Verdrängung“ zu einem Verlust des Vertrauens in die Kultur bei (ebd., 316). Kultur sei keine „Störung eines ursprünglich normal, harmonisch gewesenen und wieder harmonisch werden könnenden Lebenssystems“. Sondern umgekehrt: Ihr Grund, nicht ihre Ursache, liege in der „konstitutiven Gleichgewichtslosigkeit“ (ebd.), eben in dem Hiatus der exzentrischen Positionalität. Der Konflikt zwischen Sein und Sollen sei der Normalfall menschlicher Existenz, nicht ihre Erkrankung.

Es gebe jedoch auch „besonnene Psychoanalytiker“, die sich an die „Grenzen der Erfahrung halten" und darum wissen, dass die ganze Unterscheidung von Ich, Es und Über-Ich die Entstehung von Sitten voraussetzt, statt sie erklären zu können: „Wogegen die Philosophie Einspruch erheben muss, das ist lediglich der metaphysische Missbrauch der psychoanalytischen Gedanken“ (ebd., 318). Dieser Missbrauch sei indessen in der Annahme eines Todestriebes und eines Erlösungstriebes nun wirklich erreicht, denn diese vermeintlichen Triebe verdoppelten nur die qualitative Erfahrung des Todes und der Erlösung von Personen zu einer spekulativen Scheinerklärung dieser Erfahrungen. Diese qualitativen Erfahrungen hingegen unterstellten bereits spezifisch personale Zeitmodi der Zukunft im Unterschied zur Vergangenheit und Gegenwart (ebd., 319). Ebenso setze Freuds Zielverschiebung der Sublimierung zu geistigkulturellen Werken deren geistigen Geltungsanspruch schon voraus, statt seine Entstehung kausal erklären zu können. Man komme in einer Kausalerklärung aus den „zweckdienlichen Mitteln des Handelns“ nie in den „nichtzweckhaften Sphären der Kultivierung“ an (ebd., 320). Vielmehr müsse man anerkennen, dass man das Nicht-Zweckhafte des Geistes als Voraussetzung bereits in Anspruch nehme, wenn man zu einer Erklärung übergeht.

\subsection{Zu Plessners Einordnung und Kritik an der Psychoanalyse in seiner Abhandlung Zur Frage nach der Conditio humana (1961)}

In einem ersten konstruktiven Schritt ordnet Plessner in Zur Frage nach der Conditio humana die Triebmodelle in die Verstehensversuche der Humanontogenese und Humanphylogenese ein. Die Rekonstruktion beider Genesen brauche das „Anthropoide“ im Sinne einer Gemeinsamkeit der Vorformen von Menschen und Menschenaffen als Ausgangspunkt. Plessner schließt zunächst an 
die vergleichende Zoologie von Adolf Portmann (Portmann 1951, dazu Tolone 2015) an. In ihrem Vergleich fällt die Verlängerung des Kindheits- und Jugendstadiums als einer Zeit des Lernens und des Spielens unter Säugern, insbesondere Affen, Menschenaffen und Menschen auf. Im letzteren Falle von Menschen werde zudem auch schon die Endphase der Embryonalentwicklung ,nach außen ins Freie، verlegt, in das sogenannte ,extrauterine Frühjahr'. Statt sich durch Spezialisierung und frühes Erwachsenwerden an bestimmte Umwelten anzupassen, entstehe so zunehmend in der von der sozialen Gruppe geschützten Kindheits- und Jugendphase ein universalisierbares Lernpotential, das mehr Selbständigkeit gegenüber der Umwelt ermögliche. Vom Erben, z. B. der Erbmotorik, könne so aufs Erwerben, die Erwerbsmotorik, übergegangen werden (siehe Plessner 1983e, 165-166).

Man habe die Zerebralisierung als physisches und die Intelligenzsteigerung als psychisches Korrelat immer einseitig in einen ,Kampf ums Dasein“ eingeordnet. Man könne diese psycho-physischen Korrelate aber auch in einem kooperativen Gruppenmilieu kontextualisieren, das die Ontogenese der Nachwachsenden von der Phylogenese der Vorgänger schrittweise emanzipieren kann (siehe ebd., 167-169). Plessner denkt hier die Überwindung der biogenetischen Grundregel von Ernst Haeckel, an der sich Freud noch vielfach orientierte (Freud 1999d und Freud 1999b). Die Ontogenese sei gerade nicht auf die verkürzte Reproduktion der Phylogenese festgelegt, sondern kann in einem eigenen internen Milieu zur Emanzipation von der Phylogenese führen, das vom Spielen über das Imitieren bis zur Reziprozität und Sprachlichkeit in den soziokulturellen Interaktionen geführt haben könnte (Plessner 1983e, 172-178). In der heutigen Diskussion spricht Michael Tomasello vom ,Wagenhebereffekt ${ }^{*}$ in einer soziokulturellen Nischenbildung, der die Spezifik kultureller im Unterschied zur natürlichen Evolution freisetze (Tomasello 2002, 50-60). Der Übergang zur Kultur als einer kollektiven Intentionalität erfordere den Zwischenschritt einer zweisam geteilten Intentionalität (Tomasello 2014b).

In einem derartig sozialen Gruppenmilieu des Anthropoiden greife nun, so Plessner, eine Triebauffassung, allerdings nicht die der Psychoanalyse, sondern die aus der vergleichenden Tierpsychologie von F. J. J. Buytendijk (Buytendijk 1958a und Buytendijk 1958b, dazu Becker 2015): „In dieser Lage bildet sich ein Triebüberschuss, der nach Abfuhr drängt, aber eine unfertige Motorik und Sexualität vorfindet. Daraus resultiert das Spiel, ein zweckfreies Verhalten zwischen Bindung und Lösung, das eine von Angst und Gier unbelastete, in sich selbst erfüllte Beziehung stiftet“ (Plessner 1983e, 167). Plessner ordnet Freuds Verständnis des Spielens von Kindern noch unter die alten Theorien ein, die 
unter dem Primat des Ernstes und der Zweckmäßigkeit des erwachsenen Verhaltens stünden:

Dasselbe sagt Freud, für den ebenfalls das fiktive Element, die Ausgleichsfunktion durch Ersatzhandlungen, der Schutz des Organismus durch Entladung und Abfuhr der Triebenergie im Mittelpunkt seiner Überlegungen steht. Alle diese Theorien sehen das Spiel im Lichte des Ernstes und der Zweckmäßigkeit. Seine scheinbare Unvernunft trägt die Beweislast. (Plessner 2001c, 200)

Der „Gegenschlag zu diesen Theorien“ (ebd., 200) sei aus der Entwicklungspsychologie, der verstehenden Psychologie und Phänomenologie gekommen und neben Buytendijk in Johan Huizingas Homo ludens (Huizinga 1987) kulturhistorisch entfaltet worden. Diese neuen Theorien fragen umgekehrt danach, wie die in sich, nämlich zwischen Bindung und Lösung erfüllte Beziehung ein geistiges Potential ermöglicht, das Zwecksetzung allererst erlaubt, die sich dann auch unter Mittel-Aspekten im Äußeren ablösen lässt (Plessner 2001c, 201-202).

Ich habe ausführlich in meinem Buch Zwischen Lachen und Weinen. Bd. 1: Das Spektrum menschlicher Phänomene dieses diskontinuierliche Kontinuum vom Spielverhalten der Säuger bis zu den Dramen in der Weltgeschichte von Menschen als dem theatrum mundi umrissen (Krüger 1999, 3.6. Kap., siehe auch II. Teil im vorliegenden Band). Plessner geht es bezüglich der beiden genannten Genesen im Kern immer um den Zusammenhang zwischen Spielen in und Schauspielen mit verschiedenen Rollen. Dabei handelt es sich insbesondere um die privaten und öffentlichen Personenrollen, in deren Kontext sich für ihn auch die Sprachen und andere Symbolformen verstehen lassen, denn in ihrer Ausübung kommt es zu Bezeichnungen, die ausgelegt und interpretiert werden können. Wenn man wie Plessner mit Buytendijk die Triebverdrängungen ins Spielen und Schauspielen von Rollen einordnet, dann erscheinen sie nicht mehr per se als Erkrankung, sondern als das Erwartbarste in der Welt beider Genesen zum personalen Leben. Natürlich sei der Mensch ein Verdränger, was denn sonst? Nicht darin bestehe die Pointe, sondern in der Frage, wie er damit in den erotischen Formen des Schauspielens sein Leben meistern kann, indem er sie kultiviert und zivilisiert.

Im zweiten Schritt kommt Plessner auf den metaphysischen Missbrauch der psychoanalytischen Trieblehre zurück. Inzwischen sieht er ihn historisch vor allem in der politischen Allianz von Marxismus und Psychoanalyse vorliegen. Es handelt sich um die Wirkungsgeschichten von Marx und Freud, die sich zu Ismen ausgewachsen haben. Was für Marx und Freud, die in der bürgerlich zivilisierten Kultur aufgewachsen waren, noch als Aufklärung im „Dienst der Selbstbefreiung von Individuum und Gesellschaft gestanden“ hatte, verkehrte 
sich nun „zu einer Waffe gegen sie“ (Plessner 1983e, 161). Wirkungsgeschichtlich werden beider Werke als Erklärungen des Geistes genommen, nach denen dieser Geist als eine „Art vertrackte [...] Spiegelfechterei über ökonomischen Verhältnissen“ und „biologischen Anlagen oder Trieben“ zu begreifen sei. Dies arbeite aber „der Selbstentwertung des Menschen in die Hände“, was dem „ureigensten Humanismus“ (ebd. 160) von Marx und Freud widerspreche. Die Marxisten könnten als echte Utopisten der ursprünglichen Natürlichkeit eine essentielle Gebrochenheit im Verhältnis des Menschen zu sich nicht anerkennen. Die Utopie der verlorenen Wildform liefert, was besonders an dem üblichen Verständnis Freuds deutlich wird, wieder ein starkes Argument für die biologische Deutung der Zivilisation als eines Sündenfalls der Natur (ebd. 193).

Plessner selbst hält demgegenüber unbeirrt an dem Projekt einer transdisziplinären Rahmenwissenschaft von der Personalität des Lebens für die Fortsetzung der Zivilisationsgeschichte (siehe Krüger 2013a, im vorliegenden Band III. Teil) fest: Die Mitwelt existiere, privat und öffentlich, aus einem Geflecht von Person und Sache, einer Welt des Wir, in der jeder zu jedem in der ersten, zweiten und dritten Person singularis und pluralis die Rückbezüglichkeit und Gegenseitigkeit seiner Verhältnisse zu beachten habe (Plessner 1983e, 194), wenn diese Mitwelt nicht in Gewalt und Krieg untergehen soll.

\subsection{Die Rolle von Freuds Buch Der Witz und seine Beziehung zum Unbewussten (1905) in Plessners Monographie Lachen und Weinen (1941)}

Plessner interessiert sich in Lachen und Weinen dafür, wie man die Einsicht verstehen kann, dass im personalen Leben zwei einander entgegengesetzte Verhaltensgrenzen zum Ausdruck kommen, nämlich einerseits die Grenze im Lachen und andererseits diejenige im Weinen. Für normal würden im personalen Leben Situationen gehalten, auf die man durch Sprechen, planmäßiges Handeln und variables Gestalten antworten kann. Man weiß dabei um die Gefahr, dass man sich zu irren vermag, und hält dies für korrigierbar. „Mit dieser Gliederbarkeit, Stabilität und Beweglichkeit, einem Minimum von Eindeutigkeit und Elastizität, Ordnung und Bildsamkeit, Geschlossenheit und Offenheit rechnet das Leben“ (Plessner 1982f, 361). Wenn sich die Gefahr zu einer ernsthaften Bedrohung für Leib und Leben ausweitet, können aktuell Angst, Flucht, Abwehrhaltung entstehen, nicht aber Lachen und Weinen. Sie entstünden hingegen in Lagen, auf die die Person keine Antwort habe, in für sie unbeantwortbaren Lagen, ohne dass diese für sie aktuell bedrohlich wären. 
Dafür gebe es grundsätzlich zwei Möglichkeiten in den Richtungen personaler Verhaltensbildung: Entweder entstehe diese Unbeantwortbarkeit aus dem sich ausschließenden Widerspruch zwischen verschiedenen Beantwortungsmöglichkeiten in derselben Hinsicht, was zum Lachen tendiere, oder aus dem Sinnverlust im Ganzen, was zum Weinen tendiere. Muss sich die eine Art der Begrenzung in der Form eines Abstandnehmens von bindenden Zusammenhängen, als Loslösung, darstellen (in Spiel, Komik, Witz, Verlegenheit und Verzweiflung), so die andere Art in der Begegnung mit Losgelöstem, das unvermittelt berührt (rührt, erschüttert, verwandelt, löst, überwältigt) (ebd., 383f.).

Im ersten Fall des Lachens entfliehe die Person dem sich ausschließenden Widerspruch der Beantwortungsmöglichkeiten leiblich in die offene Welt hinaus - sie fliegt gleichsam der Situation davon. Im zweiten Fall des Weinens schließe sich die Person in die Verhältnislosigkeit der Lage ein und breche daher in ihren Leib hinein und in ihrem organismischen Körper zusammen. „Geöffnetheit, Unvermitteltheit, Eruptivität charakterisieren das Lachen, Verschlossenheit, Vermitteltheit, Allmählichkeit das Weinen. Diese Charaktere sind nicht zufällig. Der Lachende ist zur Welt geöffnet“ (ebd., 368). Der Weinende schließt sich von ihr ab. In beiden Fällen bricht das personal antwortende Zusammenspiel von Körperhaben und Leibsein auseinander, aber in entgegengesetzte Richtungen. Die Desorganisation in dem Verhältnis der Person zu ihrem Körperleib bringe zum Ausdruck, dass sie die Lage nicht beantworten kann. „Im Gegensatz zur mimischen Ausdrucksgebärde stellt sich das Genus von Lachen und Weinen als eine Äußerungsweise dar, bei welcher der Verlust der Beherrschung im Ganzen Ausdruckswert hat“ (ebd., 274).

In diesem interkulturell vergleichbaren, philosophisch-anthropologischen Untersuchungsrahmen personaler Lebensformen kommt Plessner auch auf die Anlässe des Lachens und des Weinens $\mathrm{zu}$ sprechen, die er ausdrücklich nicht als Ursachen, sondern als Verstehensgründe für den Frage- und Antwortcharakter im personalen Verhalten thematisiert. Unter den Anlässen des Lachens wird der Witz und damit Freuds Buch Der Witz und seine Beziehung zum Unbewussten (1905, hier Freud 1999c) behandelt.

Plessner würdigt Freuds Leistung problemgeschichtlich. „Zweifellos bedeutet die psychoanalytische Theorie gegenüber den Erklärungen nach formalen Prinzipien wie Verblüffung und Erleuchtung (Heymans) oder Stauung und Entladung (Lipps) einen Fortschritt“ (Plessner 1982f, 323). Plessner fasst Freuds Kerngedanken wie folgt zusammen:

Für gewöhnlich legt unsere gesellschaftliche Erziehung uns Hemmungen, Einengungen und Verdrängungen auf. Werden sie uns erspart, so empfinden wir, dass eine Last von 
uns genommen ist. Verschüttete Lustquellen aus einer Lebenszeit, in der wir seelisch mit geringerem Kraftaufwand reagierten, aus der Kindheit, werden damit wieder freigelegt. Humor erregt Lust, weil er Gefühlsaufwand, Komik, weil sie Aufwand an Vorstellungen, Witz, weil er Aufwand an Hemmungen erspart. (Ebd., 318)

Diese Hypothese werde unter drei Aspekten durchgeführt. Die Ausdruckstechnik des Witzes bestehe in seiner wesentlichen Kürze, die Worte durch Verdichtung, Verschiebung bzw. Umbiegung, Unifizierung, Darstellung des Gegenteils, Überbietung und Anspielung einspare. Der Gehalt des Witzes liege in seiner Tendenz (feindselig, obszön oder harmlos), dessen Stärke von dem „Hemmungs- und Unterdrückungsaufwand" abhängt, von dem er im Lachen befreie (ebd., 318-319). Durch diese beiden ersten Punkte gelinge es Freuds Theorie, den Witz als eine „Einheit von Ausdrucksform und Gehalt zu verstehen“ (ebd., 317). Diese Ermöglichung von Witzigkeit werde aber erst drittens in der sozialen Interaktion von Schöpfer (Erzähler) und Hörer des Witzes wirklich, wenn sich nämlich beide „ihrer wiedergewonnenen Freiheit“ im Lachen versichern (ebd., 318):

Der Traum, ein verkleideter Wunsch und in Relation zu unserem persönlichsten Lebensinteresse, ist ein asoziales Produkt mit der Tendenz der Unlustersparung. Hingegen ist der Witz ein soziales Gebilde und stellt eine Methode dar, um innerhalb der Gesellschaft, wohlgemerkt einer Gesellschaft von Erwachsenen, verlorengegangene Lustquellen zurückzugewinnen. (Ebd., 320)

Plessner hält Freuds Theorie im Hinblick auf die Fallgruppe der „tendenziösen Witze“, die von einem unbewussten Hemmungsaufwand im Sozialen befreien, für „plausibel“ (ebd.).

So sehr Plessner Freuds theoretischen Zusammenhang zwischen Ausdrucksform, Gehalt und sozialer Wirklichkeit des Witzes begrüßt, so wenig ist er von der inhaltlichen Durchführung dieses Zusammenhanges überzeugt. Freud schränke seine Analyse von Witzen auf einen unbewussten Mechanismus und auf eine Ersparung an Hemmungs- und Unterdrückungsaufwand ein. Die Kürze des Witzes müsse indessen nicht eine Ersparung sein und die Erleichterung müsse nicht aus einer unbewussten Kindheit kommen: „Kürze ist Zumutung an die Fassungskraft, somit Hemmung und Stauung, die der Hörer überwinden soll. Die Technik des verschwiegenen Verdeckens und der heimlichen Sinnverzauberung schafft erst den Widerstand, dessen Brechung sie zugleich herbeiführt. Hierin ist der Witz dem Rätsel verwandt, auf dessen Frageform er jedoch für gewöhnlich verzichtet, um eine reinere Sinnüberlegung zu erreichen. Hat man aber die Ausdruckstechnik des Witzes und der Witzigkeit als Hemmungsmittel durchschaut, dann braucht man unbewusste Hemmungen nicht zu beru- 
fen, um aus ihrer Durchbrechung den lustvollen Gewinn an frei werdendem Kraftüberschuss zu erklären. Dann ergibt sich der lustvolle Überschuss, die plötzliche Erleichterung aus dem Doppelspiel, eine Schwierigkeit zu schaffen, die sich selbst überwindet. Natürlich können Tendenzen, die in einer Gesellschaft durch Moral, Politik oder welche Umstände auch immer nicht verwirklicht werden, sich dieses Doppelspiels bedienen, und die Eruptivität des Lachens zeigt, dass in solchen Fällen noch eine besondere latente Spannung dahintersteht. Der Witz als solcher und seine Wirkung dürfen deshalb nicht aus einem unbewussten Mechanismus und nicht nach einem Sparsamkeitsprinzip begriffen werden“ (ebd., 321).

Auch für die Motive von Witz und Witzigkeit sei der Freudsche Gesichtspunkt nicht erschöpfend. Plessner anerkennt ihn, erweitert ihn aber auch auf andere Fallgruppen:

Witz kann einfach aus Freude an Beweglichkeit und Indirektheit, am Spiel von Hemmung und Lösung kommen. Er kann im Dienste feindseliger und obszöner Tendenzen stehen. Nicht weniger häufig aber ist er ein Mittel, sich von seinen Gefühlen zu befreien, sie zu verdecken oder jedenfalls so zu tun, als sei man ihrer Herr. Als Modus, von Dingen zu reden, die zu schwer und zu heikel sind, um sie im Ernst zu berühren, wird er oft bitter sein, sarkastisch, kaustisch, zynisch, vielfach ironisch, seltener humorvoll und entkrampft. Für ihn gilt Nietzsches Wort: Der Witz ist das Epigramm auf den Tod eines Gefühls. Wir fügen hinzu: ein Epigramm, das den Nagel zu seinem Sarg auf den Kopf trifft. (Ebd., 321-322)

Gerade die Fallgruppe der vielfach ironischen, sarkastischen, zynischen und kaustisch zerstörerischen Witze wirft die Frage auf, warum sie ins Lachen und nicht ins Weinen oder in Zwischenlagen von Lachen und Weinen für die Betroffenen führen: „Aber warum es gerade Lachen ist, das die Entspannung besorgt - diese Frage bleibt noch aufzuklären“ (ebd., 323), weshalb Plessner sein Thema von den Grenzerfahrungen in der personalen Lebensform wieder aufnimmt (vgl. zur Aktualität seines philosophischen Rahmens für das Lachen und Weinen Waldenfels 1994; Krüger 1999; Prütting 2013; Krüger 2017a).

\subsection{Plessners Rückgriff auf Freuds Aufsatz über Das Unheimliche (1919) in seinem Buch Macht und menschliche Natur (1931)}

Wenn die menschliche Natur in einer exzentrischen Positionalität situiert wird, dann besteht sie in der geschichtlichen Aufgabe, den Geist der exzentrisch in Anspruch genommenen Mitwelt auch in den leiblichen Konzentriken verwirklichen und in Körperbestimmungen positiv realisieren zu können. Andernfalls 
würde es sich um eine reine Geistigkeit handeln, die nicht leben kann. Der geschichtliche Lebensprozess findet in dem Widerspruch statt, Geschichte nur um den Preis machen zu können, als dass man von ihr gemacht wird. In der Geschichte stehend, erfolgt unentwegt der Versuch, alles, was geschieht, personal zurechnen zu können:

Schon Ich und Du und Wir sagen können bedeutet in der damit vollzogenen Abgrenzung der Sphären des Mein, Dein, Unser, Aller Zurechnungen, die zwar noch keinen expliziten juristischen Charakter tragen, aber eine rechtliche Ordnung des Lebens in allen seinen Äußerungen nötig machen. (Plessner 1981d, 200)

Je stabiler diese Ordnung eingerichtet wird, desto überraschender wird sie von der Geschichte den Beteiligten wieder in Frage gestellt. Es beginnt in der Generationenfolge von neuem die utopische Inanspruchnahme einer künftig besseren Mitwelt, die durch Vergegenwärtigung an Verwirklichung gegenüber dem Vergangenen gewinnt.

Plessner zieht die Konsequenz aus der Einsicht in diesen zukunftsfundierten Charakter der nie (außer im Utopischen) übergeschichtlich werdenden Existenz von personalen Lebensformen, indem er seinen geschichtlich-horizontalen Vergleich verbindlich dem Prinzip der Unergründlichkeit des Menschen, d. h. seiner offenen Fraglichkeit, unterstellt (siehe ebd., 160-164, 181-182). Damit im Vordergrund personalen Lebens positiv die ihm angemessene künstliche Umwelt gestaltet werden kann, nimmt es im Hintergrund dafür eine offene Welt als Ermöglichung in Anspruch, die von ihm nicht gleichzeitig vergegenständlicht werden kann. Gelänge letzteres, endete personales Leben in der Übernahme der Rolle Gottes. Oft wird Macht aus der überhistorischen Verallgemeinerung dessen verstanden, was man hier und jetzt im Vordergrund positiv machen kann, aber dies ändert sich ja geschichtlich gerade. Die geschichtliche Erneuerung von Macht werde nicht aus der bloßen Hochrechnung der bisherigen positiven Selbstbestimmung in personalen Zurechnungen, sondern aus der Unbestimmtheitsrelation der offenen Zukunft gegenüber der tradierten personalen Ordnung ermöglicht:

In dieser Relation der Unbestimmtheit zu sich fasst sich der Mensch als Macht und entdeckt sich für sein Leben, theoretisch und praktisch, als offene Frage. Was er sich in diesem Verzicht versagt, wächst ihm als Kraft des Könnens wieder zu. Was er an Fülle der Möglichkeiten dadurch gewinnt, gibt ihm zugleich die entschiedene Begrenzung gegen unendlich andere Möglichkeiten des Selbstverständnisses und des Weltbegreifens, die er damit schon nicht mehr hat. (Ebd., 188)

Diese wenigen Erläuterungen derjenigen Begriffe, die in dem Titel des kleinen Buchs von Plessner Macht und menschliche Natur. Ein Versuch zur Anthropologie 
der geschichtlichen Weltansicht (1931) vorkommen, müssen hier genügen (näher siehe Krüger 2013a; im vorliegenden Band Dritter Teil), um noch auf eine kurze, aber wichtige Anspielung Plessners auf Freuds Aufsatz über Das Unheimliche (1919, Freud 1999b) eingehen zu können. Es geht Plessner um eine Alterität in der Verfasstheit personalen Lebens, die nicht einfach das Andere des Gleichen ist, wie etwa das alter ego, sondern wirklich fremd wird. Diese Fremdheit werde gewöhnlich so verstanden, dass sie nur aus den ,abträglichen Wirkungen auf die eigene Sphäre der Vertrautheit“ bestehe, weshalb man vor ihr Angst habe und sich dagegen im Sinne einer aufgeklärten Zweck-Mittel-Rationalität wappne: „Aber diese Angst ist verwurzelt in der Unheimlichkeit des Fremden [...]; weil das Fremde nicht bloß ein Anderes ist“ (Plessner 1981d, 192f.). Dem aufgeklärten Menschen sei der Stein nichts Fremdes, nur anderes als er in dem Sinne der bloßen Verschiedenheit. Die Fremdheit gewinne „leise im Pflanzlichen, vernehmlicher im Tierischen Boden [...], um schließlich im Menschlichen auch noch für den aufgeklärten Menschen ihre letzte Domäne zu bekommen - und korrelativ dazu in dem rätselvollen Anblick des Universums“ (ebd.,193).

Hier folgt nun die Anspielung auf Freuds Verständnis des Unheimlichen und Plessners Interpretation seines Verweises wie folgt:

Denn das Fremde ist das Eigene, Vertraute und Heimliche im Anderen und als das Andere und darum - wir erinnern hier an eine Erkenntnis Freuds - das Unheimliche. Wenn die Formulierung erlaubt ist: Der Mensch sieht ,sich` nicht nur in seinem Hier, sondern auch im Dort des Anderen. Die Sphäre der Vertrautheit ist also nicht von ,Natur` begrenzt und erstreckt sich (gleichsam außergeschichtlich) bis zu einer gewissen Grenze, sondern sie ist offen und erschließt ihm dadurch die Unheimlichkeit des Anderen in der unbegreiflichen Verschränkung des Eigenen mit dem Anderen. (Ebd., 193)

Plessner liest hier Freud nicht kausal im Sinne des Verdrängungsmechanismus, der bewusstseinsphilosophische Annahmen unterstellt, sondern hermeneutisch, um aufzuklären, inwiefern die Verschränkung des Eigenen mit dem Anderen strukturell eine über das Eigene und Andere hinausgehende Dimension des Unheimlichen enthält, also durch Fremdheit geöffnet wird. Dafür ist Freuds Unterscheidung des Heimlichen vom Heimischen innerhalb des Eigenen der vorausgesetzte Ausgangspunkt. Schon das Eigene ist nicht auf homogene Weise in sich geschlossen, so dass es nur aus einem heimisch Vertrauten bestünde. Das Eigene enthält auch eine Heimlichkeit vor sich und anderen (so Freuds Belege aus etymologischen Wörterbüchern, siehe Freud 1999b, 232). Zur Erläuterung greift Freud auf das seit der Romantik bekannte und von E. T. A. Hoffmann verwendete Doppelgängertum (ebd., 245-249) zurück, mit dem auch Plessner arbeitet und das er bis zur „Wir-Form des eigenen Ichs“ (Plessner 1975, 
303) entfaltet hat. „Mitwelt ist die vom Menschen als Sphäre anderer Menschen erfasste Form der eigenen Position“ (ebd., 302).

Das Eigene ist nicht nur vertraut und heimisch, sondern auch heimlich vor Anderen und vor uns selbst. Das Eigene tritt nicht nur vor uns selbst auf, sondern gleichursprünglich vor Anderem. Anderes tritt nicht nur vor sich selbst auf, sondern gleichursprünglich vor uns selbst als seinem Anderen. Daher kann das Eigene wechselweise im Anderen und als das jeweils Andere des jeweils Eigenen begegnen. Dadurch wird das Heimliche unheimlich, denn es ist nicht mehr die Heimlichkeit, die im Eigenen vertraut ist, sondern die Heimlichkeit im Anderen. Dadurch ändert sich der Modus des Heimlichen ins Unheimliche. Es ist heimlich auf eine andere Art und Weise, als es die anfängliche Unterscheidung von Eigenem und Anderem vermuten ließ, da es eben unheimlich geworden ist. Diese Art und Weise, die anders als die Ausgangsunterscheidung des Eigenen vom Anderen ist, ist fremd, denn sie wird wechselseitig für das Eigene und das Andere unheimlich. Die Fremdheit in der Verschränkung des Eigenen mit dem Anderen ist den davon Betroffenen unbegreiflich. Die Öffnung der Ausgangsunterscheidung zwischen Eigenem und Anderem durch Fremdheit ist ambivalent, was Freud für heimlich-unheimlich ausdrücklich vermerkt (Freud 1999b, 235) und Plessner an seiner Erkenntnis würdigt, denn sie passt zu Plessners Einsicht in die ontologische Zweideutigkeit in der Individualisierung von Personen (siehe 5. Kapitel im vorliegenden Band).

Aber Plessner braucht hier die Ambivalenz der Fremdheit nur als das strukturell Unheimliche, das zu einer Quelle von eskalierender Angst werden kann, die nicht einfach kreatürlich ist, sondern auf einem inter- und intra-personalen Niveau liegt. An anderen grundsätzlichen Stellen würdigt Plessner die andere Seite in der Ambivalenz von Fremdheit, die auch eine „seelige Fremde“ (Plessner 1975, 346) sein könne, wenn sie vom Eigenen befreie. Plessner vermerkt hier auch ausdrücklich, dass das Unheimliche nicht mit dem Feindlichen gleichzusetzen sei (Plessner 1981d, 195). Diese Gleichsetzung erfolge aber in der von Carl Schmitt vertretenen Universalisierung von Politik. Die Bindung und Entfaltung von existenzieller Angst geschehe dann durch den Gegensatz, sich zwischen entweder Freund oder Feind entscheiden zu müssen. Die Ambivalenz der Fremdheit und ihre kurzschlüssigen Abschaffungsversuche in diktatorischen Bewegungen sind ein wirkliches Problem im Geschichtsprozess, das man nicht so leicht den Autoren anlasten kann, die sich mit ihm beschäftigen. Es erledigt sich nicht dadurch, dass man Plessner eine zu große Nähe zu Schmitt vorwirft (Waldenfels 1997, 48), was hier aber nicht mehr näher verfolgt werden kann (siehe Krüger 2013a und im vorliegenden Band den III. Teil). 
Wir haben damit einen Überblick über die vier wichtigsten, weil systematisch relevanten Stellungnahmen aus Plessners Philosophischer Anthropologie zu Freuds klassischer Psychoanalyse von den 1920er bis in die 1960er Jahren hinein gewonnen. Um sie richtig verstehen zu können, war es zunächst nötig, Plessners Vorschlag für eine philosophisch-anthropologische Rahmenkonzeption von den horizontalen und vertikalen Relationen der personal geteilten Mitwelt in Erinnerung zu rufen.

Einerseits traf der naturwissenschaftlich-medizinische Erklärungsanspruch der klassischen Psychoanalyse auf die naturphilosophisch-vertikale Fundierung der personalen Lebensform in der Philosophischen Anthropologie. In dieser Hinsicht besagte Plessners Kritik, dass der Psychoanalyse die Spezifikation des personalen Niveaus menschlicher Existenz fehle, sowohl vertikal, was das Verhältnis der Personalität zu ihrer Körper-Leib-Differenz angeht, als auch horizontal, was die Relation zwischen Personen betrifft, die als private und öffentliche Doppelgänger existieren. Die Trieblehre könne schon auf Primaten zutreffen, die keine Personalität in Gemeinschafts- und Gesellschaftsrollen ausbilden. Durch dieses philosophische Defizit könne die Psychoanalyse als eine Metaphysik und/oder politisch-ideologisch missbraucht werden. Für ihre Neubegründung müsste sie sich der Konzeption eines freien, in sich erfüllten Spiels zwischen Bindung und Lösung der Bindung (Buytendijk/Huizinga) öffnen, an die eine Schauspieltheorie der personalen Lebensform (Plessner) anschließen kann. Damit entfalle die biogenetische Grundregel, nach der die Ontogenese nur eine verkürzte Wiederholung der Phylogenese sei, unter deren Eindruck Freud noch seinen kausalen Mechanismus als Erklärung entworfen habe.

Andererseits bestätigt Plessners Freud-Lektüre, dass Freud auch als kulturwissenschaftlicher Hermeneutiker gelesen werden kann, der dabei hilft, phänomenbezogene Einsichten in die conditio humana verstehen zu können. Dies hat sich in Plessners Würdigung von Freuds Analyse der Witzigkeit gezeigt, zählen doch Witze zu den Anlässen des Lachens, die Plessner symmetrisch zu den Anlässen des Weinens als den Grenzerfahrungen in der personalen Lebensform untersucht hat. Dies hat sich auch in Plessners Verwendung von Freuds Erkenntnis des Unheimlichen erwiesen. Freuds Aufdeckung der Ambivalenz im Phänomenpaar ,heimlich-unheimlich` und ihre Verdeutlichung durch das Doppelgängertum im Menschsein passt zu Plessners Einsicht in die ontologische Zweideutigkeit der Individualisierung von Personen in inter- und intrapersonalen Relationen.

Während in der naturphilosophisch-vertikalen Richtung für Plessner die Kritik an Freuds naturwissenschaftlich-medizinisch kausalem Erklärungsanspruch im Vordergrund steht, um überhaupt das personale Niveau der Lebens- 
form von Menschen als Maßstab des wissenschaftlichen Fundaments zu sichern, stehen in der geschichtsphilosophisch-horizontalen Richtung die Würdigung und Integration von Freuds phänomenbezogenen Einsichten und hermeneutischen Verstehensvorschlägen im Vordergrund. Man darf hoffen, dass es in den aktuellen Versuchen zur Neubegründung einer transdisziplinären Rahmenwissenschaft von der Personalität endlich zu einem produktiven Dialog zwischen der Philosophischen Anthropologie und der Psychoanalyse kommen wird, hat letztere doch längst durch ihre nachklassische Entwicklung mit der biogenetischen Grundregel und bloßen Trieblehren gebrochen. 